\title{
Tau-function formalism for supersymmetric KP hierarchies
}

\author{
Luis Martínez Alonso and Elena Medina Reus \\ Departamento de Fisica Teórica, Universidad Complutense, E28040 Madrid, Spain
}

(Received 20 March 1995; accepted for publication 21 April 1995)

\begin{abstract}
We consider the Manin-Radul and Jacobian supersymmetric KP hierarchies from the point of view of the tau-function formalism. Solutions of their associated systems of Sato equations are characterized in terms of correlation functions of supersymmetric vertex operators of superghost type. The expression of the wave functions of these hierarchies in terms of tau-functions is obtained and the corresponding bilinear identities are established. Explicit methods for generating soliton and rational solutions are given. (0) 1995 American Institute of Physics.
\end{abstract}

\section{INTRODUCTION}

The purpose of this article is to provide a tau-function formalism for supersymmetric KP hierarchies (SKP) by using an appropriate generalization of the operator methods of the Kyoto school. ${ }^{1}$ The experience with the standard KP hierarchy shows that this formalism is relevant not only in the context of the theory of integrable systems but also from the point of view of important aspects of two-dimensional quantum field theory as, for example, the analysis of the double scaling limit $^{2}$ in the matrix-model approach to two-dimensional gravity. ${ }^{3}$ In this sense, it is likely that the two SKP hierarchies considered in this article, namely, the hierarchy formulated by Manin and Radul (MRKP) ${ }^{4}$ and the Jacobian hierarchy of Mulase and Rabin (JKP), ${ }^{5,6}$ underly some models in supergravity, ${ }^{7-9}$ as they have been shown to admit additional symmetries of superVirasoro type. ${ }^{10}$

The present work is based on the analysis of the correlation functions determined by a superghost system on the complex plane and its corresponding GL $(\infty \mid \infty)$ supergroup. These quantum fields are expressed in terms of the supersymmetric bosonization of Martinec and Sotkov, ${ }^{11}$ and the vertex operator representation of LeClair. ${ }^{12}$ An essential point in our study is the description of the analytic properties of correlation functions with the help of $\bar{\partial}$-equations. This allows us to establish the link between the superghost system and the SKP hierarchies by proving that suitable correlation functions are solutions of a $\bar{\partial}$-equation, which in turn represents an inverse scattering problem for the SKP hierarchies.

In order to illustrate the relationship between the operator method of the Kyoto school and the $\bar{\partial}$ formalism, ${ }^{13-15}$ we devote Sec. II to describe the main aspects of the theory of the KP hierarchy from this point of view and to prepare the strategy for the analysis of its supersymmetric versions. The first part of Sec. III deals with the notion of wave function for SKP hierarchies and, in particular, it includes the derivation of the expression of the vacuum wave function as well as the formulation of a relevant $\bar{\partial}$-equation. The second part of Sec. III contains the description of the operator method for SKP hierarchies. Thus, we show how to generate solutions of SKP hierarchies from deformations of correlation functions and, as a consequence, how the tau-function enters into play. Our main results are the following:

(1) Wave functions are characterized in terms of tau-functions and the corresponding bilinear identities are obtained.

(2) The expression of the tau-function as a correlation function involving the elements of the supergroup GL $(\infty \mid \infty)$ is given.

(3) General methods for obtaining solutions of the SKP hierarchies are provided. 


\section{II. $\bar{\partial}$-EQUATIONS AND QUANTUM FIELDS IN THE KP HIERARCHY}

\section{A. Wave functions for the KP hierarchy}

The theory of the KP hierarchy can be described through the algebra $\mathscr{E}$ of pseudodifferential operators

$$
X=\sum_{n \leqslant N} a_{n}(x, \mathrm{t}) \partial^{n}, \quad \partial=\frac{\partial}{\partial x},
$$

where the coefficients $a_{n}$ are elements of the algebra $\not$ of formal power series on a variable $x$ and an infinite set of time parameters $t=\left\{t_{n}\right\}_{n \geqslant 1}$. Any operator $X$ has a decomposition $X=X_{+}+X_{-}$, with $X_{+}$being the differential operator part of $X$. The flows of the KP hierarchy are determined by an infinite system of Sato equations

$$
\frac{\partial K}{\partial t_{n}}=-\left(K \partial^{n} K^{-1}\right)-K, \quad n \geqslant 1
$$

for a pseudodifferential operator

$$
K=1+\sum_{n=1}^{\infty} w_{n}(x, \mathrm{t}) \partial^{-n}
$$

A wave functions of the KP hierarchy is a function $f=f(z, x, t)$, where $z$ is a spectral parameter, which satisfies two properties: firstly, it is a solution of an infinite system of equations of the form

$$
\frac{\partial f}{\partial t_{n}}=P_{n} f, \quad n \geqslant 1,
$$

with $P_{n}$ being differential operators $\left(P_{n}=\left(P_{n}\right)_{+}\right)$in $\mathscr{E}$, and, secondly, as $z \rightarrow \infty$ it has an asymptotic expansion

$$
f=\left(1+\sum_{n=1}^{\infty} w_{n} z^{-n}\right) f_{0}
$$

where

$$
f_{0} \equiv \exp \left[\sum_{n=1}^{\infty} t_{n} \partial^{n}\right] e^{z x}=\exp \left[z x+\sum_{n=1}^{\infty} z^{n} t_{n}\right] .
$$

The coefficients $w_{n}$ of the asymptotic expansion of a wave function determine a solution $K$ of Eq. (1). Indeed, let us define $K$ by means of the relationship

$$
f=K f_{0}, \quad z \rightarrow \infty,
$$

where it is assumed that

$$
\partial^{-1} f_{0}=z^{-1} f_{0}
$$

Then Eq. (2) implies 


$$
\frac{\partial K}{\partial t_{n}}+K \partial^{n}+P_{n} K, \quad n \geqslant 1
$$

so that

$$
P_{n}=\left(K \partial^{n} K^{-1}\right)+
$$

and therefore

$$
\frac{\partial K}{\partial t_{n}}=-K \partial^{n}+P_{n} K=-\left(K \partial^{n} K^{-1}\right)_{-} K
$$

A general method for constructing wave functions of integrable systems is based on the consideration of $\bar{\partial}$ equations ${ }^{13-15}$

$$
\frac{\partial}{\partial \bar{z}} F(z, x, \mathrm{t})=\int d^{2} z^{\prime} a\left(z^{\prime}, z\right) F\left(z^{\prime}, x, \mathrm{t}\right),
$$

where $d^{2} z^{\prime}=d\left(\operatorname{Re} z^{\prime}\right) \cdot d\left(\operatorname{Im} z^{\prime}\right)$ and $a\left(z^{\prime}, z\right)$ is a given distribution. Let $\mathscr{F}$ be the space of solutions $F$ of Eq. (5) which as $z \rightarrow \infty$ have an asymptotic expansion of the form

$$
F=\left(\sum_{n=-\infty}^{N} u_{n} z^{n}\right) f_{0} .
$$

Since the integral kernel $a\left(z^{\prime}, z\right)$ of Eq. (5) does not depend on $t$ (isospectrality property), the space $\mathscr{F}$ is a module for the algebra of differential operators generated by $\partial$ and $\partial / \partial t_{n}(n \geqslant 1)$ with coefficients depending on $(x, t)$. Thus, provided that a unique solution of Eq. (5) satisfying Eq. (3) exists, such a solution will also satisfy a system of equations of the form (2) and, therefore, it will be a wave function. In this sense, it is convenient to introduce the modified wave function $\hat{f} \equiv f \cdot f_{0}^{-1}$, which is regular at infinity $(\hat{f} \rightarrow 1$ as $z \rightarrow \infty)$ and satisfies a $\bar{\partial}$-equation of the form (5) with an integral kernel given by

$$
\hat{a}\left(z^{\prime}, z, x, \mathrm{t}\right)=a\left(z^{\prime}, z\right) \frac{f_{0}\left(z^{\prime}, x, \mathrm{t}\right)}{f_{0}(z, x, \mathrm{t})} .
$$

Consequently, $\hat{f}$ satisfies the integral equation

$$
\hat{f}(z, x, \mathrm{t})=1+\frac{1}{\pi} \int d^{2} z^{\prime} d^{2} z^{\prime \prime} \frac{1}{z-z^{\prime}} \hat{a}\left(z^{\prime \prime}, z^{\prime}, x, \mathrm{t}\right) \hat{f}\left(z^{\prime \prime}, x, \mathrm{t}\right) .
$$

It is easy to see that under simple choices of the distribution $a\left(z^{\prime}, z\right)$, Eq. (6) reduces to uniquely solvable algebraic equations.

\section{B. Fermion fields and $\tau$-functions}

Since the work of the Kyoto school ${ }^{1}$ we know that the KP hierarchy is deeply related with a $b-c$ system of fermion fields on the complex plane. These fields satisfy the anticommutation relations

$$
\left\{b(z), b\left(z^{\prime}\right)\right\}=\left\{c(z), c\left(z^{\prime}\right)\right\}=0, \quad\left\{b(z), c\left(z^{\prime}\right)\right\}=\hat{\delta}\left(z-z^{\prime}\right),
$$

where $\hat{\delta}$ is the delta function on a given circle $\gamma(|z|=r)$ 


$$
\frac{1}{2 \pi i} \oint_{\gamma} d z \hat{\delta}(z-w) \varphi(z)=\varphi(w)
$$

An explicit representation is provided by the bosonization formulas

$$
b(z)=: e^{\Phi(z)}:, \quad c(z)=: e^{-\Phi(z)},
$$

where

$$
\Phi(z)=\ln u+\sum_{n=1}^{\infty} z^{n} x_{n}+\ln z u \frac{\partial}{\partial u}-\sum_{n=1}^{\infty} \frac{1}{n z^{n}} \frac{\partial}{\partial x_{n}} .
$$

Here, $u$ is a complex variable and $x=\left\{x_{n}\right\}_{n \geqslant 1}$ denotes an infinite set of complex variables. The symbol : : stands for normal ordering with respect to the bosonic variables.

The fields $b(z)$ and $c(z)$ act on a Fock space generated by the monomials

$$
|n, N\rangle=u^{n} x^{N}
$$

where $x^{N}=x_{1}^{N_{1}} x_{2}^{N_{2}} \ldots$, with $N_{i}$ being positive integers. The inner product is determined by

$$
\left\langle n, N \mid n^{\prime}, N^{\prime}\right\rangle=\delta_{n n^{\prime}} \cdot \delta_{N N^{\prime}} \cdot \frac{N_{1} ! N_{2} ! \cdots}{1^{N_{1}} 2^{N_{2} \ldots}} .
$$

As a consequence, $u^{\dagger}=u^{-1}, x_{n}^{\dagger}=(1 / n)\left(\partial / \partial x_{n}\right)$. The vacuum vector is given by $|0\rangle=1$.

The two-point function for the field $\Phi$ is

$$
\left\langle 0\left|T\left[\Phi(z) \Phi\left(z^{\prime}\right)\right]\right| 0\right\rangle=\ln \left(z-z^{\prime}\right),
$$

where $T$ stands for the time ordering of operators with respect to Euclidean time $t=\ln |z|$. Hence, for the $b-c$ system we deduce

$$
\begin{gathered}
\left\langle 0\left|T\left[b(z) b\left(z^{\prime}\right)\right]\right| 0\right\rangle=\left\langle 0\left|T\left[c(z) c\left(z^{\prime}\right)\right]\right| 0\right\rangle=0, \\
\left\langle 0\left|T\left[b(z) c\left(z^{\prime}\right)\right]\right| 0\right\rangle=\frac{1}{z-z^{\prime}} .
\end{gathered}
$$

The essential point in the relationship between the $b-c$ system and the KP theory is that the propagator of $b$ and $c$ is the Green function of the $\bar{\partial}$ operator

$$
\frac{\partial}{\partial \bar{z}}\left\langle 0\left|T\left[b(z) c\left(z^{\prime}\right)\right]\right| 0\right\rangle=\pi \delta\left(z-z^{\prime}\right),
$$

where $\delta\left(z-z^{\prime}\right)$ is the distribution

$$
\int d^{2} z \delta\left(z-z^{\prime}\right) \varphi(z)=\varphi\left(z^{\prime}\right)
$$

In this way we may construct solutions of Eq. (5) from appropriate correlation functions. Thus, given a distribution $a\left(z^{\prime}, z\right)$, let us consider

$$
F(z, x, \mathrm{t})=\langle 1|\exp [H(x, \mathrm{t})] T[b(z) g]| 0\rangle,
$$

where $|1\rangle=u, H$ is the operator 


$$
H=x \frac{\partial}{\partial x_{1}}+\sum_{n=1}^{\infty} t_{n} \frac{\partial}{\partial x_{n}}
$$

and $g$ is the following element of the group GL( $\infty)$

$$
g=\exp A, \quad A=-\frac{1}{\pi} \int d^{2} p d^{2} q b(p) a(p, q) c(q) .
$$

By using Wick's theorem, the operator

$$
T[b(z) g]=\sum_{N=0}^{\infty} \frac{1}{N !} T\left[b(z) A^{N}\right]
$$

can be expanded as a series of normal ordercd terms with all possible $T$ pairings. Moreover, the only nonanalytic terms in $z$ come from the pairings of $b(z)$ with the operators $c\left(z^{\prime}\right)$ inside the factors $A$, so that by taking Eq. (8) into account it is easy to deduce that Eq. (9) satisfies Eq. (5). On the other hand, from Eqs. (4), (7) and since as $z \rightarrow \infty$ we have $T[b(z) g]=b(z) T[g]$, the asymptotic form of Eq. (9) is

$$
F(z, x, \mathrm{t})=\tau(x, \mathrm{t}-\epsilon(z)) f_{0}(z, x, \mathrm{t}),
$$

where the function $\tau$ is defined by

$$
\tau(x, \mathrm{t})=\langle 0|\exp [H(x, \mathrm{t})] T[g]| 0\rangle,
$$

the symbol $\epsilon(z)=\left\{\epsilon_{n}(z)\right\}$ denotes the vector

$$
\epsilon_{n}(z)=\frac{1}{n z^{n}}
$$

and $f_{0}$ is the function defined in Eq. (4). To deduce this expression observe that

$$
\begin{aligned}
\langle 1|\exp [H(x, \mathrm{t})] b(z) T[g]| 0\rangle= & \langle 0| \exp \left(z x+\sum_{n=1}^{\infty} z^{n}\left(x_{n}+t_{n}\right)\right) \\
& \left.\times \exp \left(x \frac{\partial}{\partial x_{1}}+\sum_{n=1}^{\infty}\left(t_{n}-\epsilon_{n}(z)\right) \frac{\partial}{\partial x_{n}}\right)\right) T[g]|0\rangle \\
= & f_{0}(z, x, t)\langle 0|\exp [H(x, \mathrm{t}-\epsilon(z))] T[g]| 0\rangle .
\end{aligned}
$$

The above properties imply that

$$
f(z, x, \mathrm{t})=\frac{F(z, x, \mathrm{t})}{\tau(x, \mathrm{t})}
$$

is a wave function for the KP hierarchy. Indeed, from Eq. (10) it follows that as $z \rightarrow \infty$ Eq. (12) has an asymptotic expansion of the form (3). On the other hand, Eq. (12) satisfies Eq. (5).

Observe that in view of Eq. (10) as $z \rightarrow \infty$ the wave function (12) can be written in terms of the $\tau$-function as

$$
f(z, x, \mathrm{t})=\frac{\tau(x, \mathrm{t}-\epsilon(z))}{\tau(x, \mathrm{t})} f_{0}(z, x, \mathrm{t})
$$


The bilinear identity for the KP hierarchy derives easily from the consideration of the dual wave function, which can be constructed from the correlation function

$$
G(z, x, \theta, \mathrm{t})=\langle-1|\exp [H(x, \mathrm{t})] T[c(z) g]| 0\rangle,
$$

where $|-1\rangle=u^{-1}$. Then, by repeating the arguments used in the analysis of $F$ one deduces the following $\bar{\partial}$-equation for $G$ :

$$
\frac{\partial}{\partial \bar{z}} G(z, x, \mathrm{t})=-\int d^{2} z^{\prime} a\left(z, z^{\prime}\right) G\left(z^{\prime}, x, \mathrm{t}\right)
$$

Thus the dual wave function may be defined by

$$
g(z, x, \mathrm{t})=\frac{G(z, x, \mathrm{t})}{\tau(x, \mathrm{t})}
$$

and as $z \rightarrow \infty$ its asymptotic form is

$$
g(z, x, \mathrm{t})=\frac{\tau(x, \mathrm{t}+\epsilon(z))}{\tau(x, \mathrm{t})}\left(f_{0}(z, x, \mathrm{t})\right)^{-1}
$$

If we take a circle $\mathcal{\gamma}(|z|=r)$ in the complex plane such that the support of the distribution $a(p, q)$ lies inside $\gamma \times \gamma$, the following bilinear identity holds:

$$
\oint_{\gamma} d z f(z, x, \mathrm{t}) g\left(z, x^{\prime}, \mathrm{t}^{\prime}\right)=0
$$

To prove Eq. (17) note that Eqs. (5) and (14) imply

$$
\begin{aligned}
\oint_{\gamma} d z f(z, x, t) g\left(z, x^{\prime}, t^{\prime}\right)= & 2 i \int d^{2} z \frac{\partial}{\partial \bar{z}}\left[f(z, x, t) g\left(z, x^{\prime}, t^{\prime}\right)\right] \\
= & 2 i \int d^{2} z d^{2} z^{\prime} f\left(z^{\prime}, x, t\right) a\left(z^{\prime}, z\right) g\left(z, x^{\prime}, t^{\prime}\right) \\
& -2 i \int d^{2} z d^{2} z^{\prime} f(z, x, t) a\left(z, z^{\prime}\right) g\left(z^{\prime}, x^{\prime}, t^{\prime}\right)=0 .
\end{aligned}
$$

By inserting Eqs. (13) and (16) into Eq. (17) we get the usual expression of the bilinear identity of the KP hierarchy in terms of the $\tau$-function

$$
\oint_{\gamma} d z f_{0}(z, x, \mathrm{t}) f_{0}^{-1}\left(z, x^{\prime}, \mathrm{t}^{\prime}\right) \tau(x, \mathrm{t}-\epsilon(z)) \tau\left(x^{\prime}, \mathrm{t}+\epsilon(z)\right)=0 .
$$

\section{III. $\bar{\partial}$-EQUATIONS AND QUANTUM SUPERFIELDS IN SUPER KP HIERARCHIES}

\section{A. Wave functions for super-KP hierarchies}

The MRKP and JKP hierarchies can he described in a unified way with the help of an algebra $\hat{\mathscr{E}}$ of superpseudodifferential operators

$$
X=\sum_{n \leqslant N} a_{n}(x, \theta, \mathrm{t}) D^{n}
$$


where the coefficients $a_{n}$ are elements of a supercommutative algebra $\hat{A}$ of superfunctions depending on an even variable $x$, an odd variable $\theta$, and an infinite set of time parameters $t=\left\{t_{n}\right\}_{n \geqslant 1}$ which includes even and odd variables $t_{2 n}$ and $t_{2 n-1}$, respectively. The symbol $D$ is defined as the derivation on $\hat{A}$ given by

$$
D=\frac{\partial}{\partial \theta}+\theta \frac{\partial}{\partial x}
$$

We will denote by $X=X_{+}+X_{-}$the decomposition of elements $X \in \hat{\mathscr{E}}$ into positive and negative powers of $D$.

The Sato equations for the super-KP (SKP) hierarchies can be written as

$$
D_{n} K=-\left(K A_{n} K^{-1}\right)_{-} K, \quad n \geqslant 1
$$

for an even $K \in \hat{\mathscr{E}}$ of the form

$$
K=1+\sum_{n=1}^{\infty} w_{n}(x, \theta, \mathrm{t}) D^{-n}
$$

The coefficients of $K$ are assumed to have parity $p\left(w_{2 n}\right)=0$ and $p\left(w_{2 n+1}\right)=1$ so that $p(K)=0$.

For the MRKP hierarchy the superderivations $D_{n}$ and the operators $A_{n}$ in Eq. (19) are

$$
D_{2 n}=\frac{\partial}{\partial t_{2 n}}, \quad D_{2 n-1}=\frac{\partial}{\partial t_{2 n-1}}-\sum_{m \geqslant 1} t_{2 m-1} \frac{\partial}{\partial t_{2 n+2 m-2}}, \quad A_{n}=D^{n},
$$

while for the JKP version

$$
D_{n}=\frac{\partial}{\partial t_{n}}, \quad A_{2 n}=D^{2 n}, \quad A_{2 n-1}=\left(D-\theta D^{2}\right) D^{2 n-2}
$$

In order to introduce the notion of wave function for a SKP hierarchy we first define the vacuum wave function ${ }^{16,6-10} f_{0}$ as

$$
f_{0}=V e^{z x+\alpha \theta},
$$

where $V$ is the element of $\hat{\mathscr{E}}$ verifying

$$
D_{n} V=A_{n} V,\left.\quad V\right|_{t=0}=1, \quad n \geqslant 1 .
$$

Thus, for the MRKP hierarchy we have

$$
\begin{gathered}
V=\exp \left[\sum_{n=1}^{\infty} t_{2 n} D^{2 n}\right] \cdot\left[1+\sum_{n=1}^{\infty} t_{2 n-1} D^{2 n-1}\right], \\
f_{0}=\exp \left[z x+\alpha \theta+\sum_{n=1}^{\infty} z^{n} t_{2 n}+(\alpha-z \theta) \sum_{n=1}^{\infty} z^{n-1} t_{2 n-1}\right]
\end{gathered}
$$

and for the JKP hierarchy we get

$$
V=\exp \left[\sum_{n=1}^{\infty} t_{2 n} D^{2 n}+\sum_{n=1}^{\infty} t_{2 n-1} \frac{\partial}{\partial \theta} D^{2 n-2}\right],
$$




$$
f_{0}=\exp \left[z x+\alpha \theta+\sum_{n=1}^{\infty} z^{n} t_{2 n}+\alpha \sum_{n=1}^{\infty} z^{n-1} t_{2 n-1}\right] \text {. }
$$

Henceforth we will write both expressions of $f_{0}$ as

$$
f_{0}=\exp \left[z x+\sum_{n=1}^{\infty} z^{n}\left(t_{2 n}+\theta t_{2 n-1}\right)+(\alpha-\lambda)\left(\theta+\sum_{n=1}^{\infty} z^{n-1} t_{2 n-1}\right)\right]
$$

where $\lambda=\lambda(z, \theta, t)$ denotes a function which for the MRKP hierarchy is

$$
\lambda=z \theta-\sum_{n=1}^{\infty} z^{n} t_{2 n-1}
$$

and for the JKP hierarchy is given by

$$
\lambda=z \theta
$$

We may now define a wave function as a superfunction $f=f(Z, x, \theta, t)$ depending on a superspectral parameter $Z=(z, \alpha)(p(z)=0, p(\alpha)=1)$, which satisfies an infinite system of equations

$$
D_{n} f=P_{n} f, \quad n \geqslant 1,
$$

with $P_{n} \in \hat{\mathscr{E}}$ being differential superoperators $\left(P_{n}=\left(P_{n}\right)_{+}\right)$, and as $z \rightarrow \infty$ has an asymptotic expansion of the form

$$
f=\left(1+\sum_{n=1}^{\infty} w_{2 n} z^{-n}+(\alpha-\lambda) \sum_{n=1}^{\infty} w_{2 n-1} z^{-n}\right) f_{0}
$$

where the coefficients $w_{n}$ are assumed to be elements of $\hat{\mathscr{B}}$.

We notice that

$$
D f_{0}=(\lambda-\alpha) f_{0}, \quad D^{2} f_{0}=z f_{0}
$$

so that

$$
D^{-1} f_{0}=D \cdot D^{-2} f_{0}=(\lambda-\alpha) z^{-1} f_{0}
$$

From Eqs. (22) and (23) it is now easy to prove that a wave function determines a solution $K$ of Eq. (19) by means of the relationship

$$
f=K f_{0}, \quad z \rightarrow \infty .
$$

We may use $\bar{\partial}$-equations to determine wave functions for SKP hierarchies. They can be written in the form

$$
\frac{\partial}{\partial \bar{z}} F(Z, x, \theta, \mathrm{t})=\int d^{2 \mid 1} Z^{\prime} a\left(Z^{\prime}, Z\right) F\left(Z^{\prime}, x, \theta, \mathrm{t}\right)
$$

where $d^{2 \mid 1} Z^{\prime}=d^{2} z^{\prime} d \alpha^{\prime}$ and $a\left(Z^{\prime}, Z\right)$ is a given distribution with odd parity, which depends on $Z=(z, \alpha)$ and $Z^{\prime}=\left(z^{\prime}, \alpha^{\prime}\right)$. Let $\hat{\mathscr{F}}$ be the space of solutions $F$ of Eq. (24) which as $z \rightarrow \infty$ have an asymptotic expansion of the form 


$$
F=\left(\sum_{n=-\infty}^{N} u_{n} z^{n}+(\alpha-\lambda) \sum_{n=-\infty}^{M} v_{n} z^{n}\right) f_{0},
$$

with coefficients $u_{n}, v_{n} \in \hat{\mathscr{C}}$. By taking into account the properties of the vacuum wave function $f_{0}$ and the isospectrality of Eq. (24), it is straightforward to prove that $\hat{\mathscr{F}}$ is a left module for the algebra of superdifferential operators generated by $D$ and $D_{n},(n \geqslant 1)$ with coefficients in the algebra of superfunctions $A$. In this way, if a unique even solution $f$ of Eq. (24) with the asymptotic expansion (23) exists, then it will be a wave function for the corresponding SKP hierarchy.

We may reduce Eq. (24) to an integral equation by introducing the modified wave function

$$
\hat{f} \equiv e^{\lambda(\partial / \partial \alpha)}\left[f \cdot f_{0}^{-1}\right] \text {. }
$$

Note that $\hat{f} \rightarrow 1$ as $z \rightarrow \infty$. Moreover, $\hat{f}$ satisfies a $\bar{\partial}$-equation of the form (24) with an integral kernel given by

$$
\hat{a}\left(Z^{\prime}, Z, x, \theta, \mathrm{t}\right)=e^{\lambda(\partial / \partial \alpha)+\lambda^{\prime}\left(\partial / \partial \alpha^{\prime}\right)} a\left(Z^{\prime}, Z\right) \frac{f_{0}\left(Z^{\prime}, x, \theta, \mathrm{t}\right)}{f_{0}(Z, x, \theta, \mathrm{t})},
$$

where $\lambda^{\prime}=\lambda\left(z^{\prime}, \theta, t\right)$. Hence

$$
\hat{f}(Z, x, \theta, \mathrm{t})=1+\frac{1}{\pi} \int d^{2 \mid 1} Z^{\prime} d^{2 \mid 1} Z^{\prime \prime} \frac{\alpha-\alpha^{\prime}}{z-z^{\prime}} \hat{a}\left(Z^{\prime \prime}, Z^{\prime}, x, \theta, \mathrm{t}\right) \hat{f}\left(Z^{\prime \prime}, x, \theta, \mathrm{t}\right) .
$$

\section{B. Quantum superfields and tau-functions}

We are going to characterize solutions of Eq. (24) from correlation functions of a $B-C$ superghost system with commutation and anticommutation relations

$$
\left[B(Z), B\left(Z^{\prime}\right)\right]=\left\{C(Z), C\left(Z^{\prime}\right)\right\}=0, \quad\left[B(Z), C\left(Z^{\prime}\right)\right]=\left(\alpha-\alpha^{\prime}\right) \hat{\delta}\left(z-z^{\prime}\right),
$$

where $\hat{\delta}$ denotes the delta function on a circle $\gamma$. The supcrsymmetric bosonization of Martinec-Sotkov ${ }^{11,12}$ allows us to write these fields as

$$
B(Z)=: e^{\Phi(Z)} ;, \quad C(Z)=: e^{-\Phi(Z)} D_{z} \bar{\Phi}(Z):
$$

where $D_{z}=(\partial / \partial \alpha)+\alpha(\partial / \partial z)$, and

$$
\Phi(Z)=\ln u+\sum_{n=1}^{\infty} z^{n} x_{2 n}+\alpha \sum_{n=1}^{\infty} z^{n-1} x_{2 n-1}+\ln z \bar{u} \frac{\partial}{\partial \bar{u}}-\sum_{n=1}^{\infty} \frac{1}{n z^{n}} \frac{\partial}{\partial \bar{x}_{2 n}}+\sum_{n=1}^{\infty} \frac{\alpha}{z^{n}} \frac{\partial}{\partial \bar{x}_{2 n-1}} .
$$

The expression for $\bar{\Phi}(Z)$ is obtained from $\Phi(Z)$ under the replacement $\left(u, \bar{u}, x_{n}, \bar{x}_{n}\right) \rightarrow\left(\bar{u}, u, \bar{x}_{n}, x_{n}\right)$. Here, $u$ and $\bar{u}$ are two bosonic variables and $\mathrm{x}=\left\{x_{n}\right\}_{n \geqslant 1}$ and $\mathrm{x}^{-}=\left\{\bar{x}_{n}\right\}_{n \geqslant 1}$ denote two infinite sets of bosonic and fermionic variables $\left(p\left(x_{2 n}\right)=p\left(\bar{x}_{2 n}\right)=0\right.$, $\left.p\left(x_{2 n-1}\right)=p\left(\bar{x}_{2 n-1}\right)=1\right)$.

The corresponding super Fock space is generated by the monomials

$$
|n, \bar{n}, N, \bar{N}, M, \bar{M}\rangle=u^{n} \bar{u}^{\bar{n}} \mathrm{x}_{0}^{N} \mathrm{x}_{1}^{M} \overline{\mathrm{x}}_{0}^{\bar{N}} \overline{\mathrm{X}}-\bar{M},
$$

where $\mathrm{x}_{0}^{N}=x_{2}^{N_{1}} x_{4}^{N_{2}} \cdots, \mathrm{x}_{1}^{M}=x_{1}^{M_{1}} x_{3}^{M_{2}} \cdots$, and so on, with $N_{i}$ being positive integers and $M_{i}=0$ or 1 . The inner product in this space is defined by assuming orthogonality for any pair of different monomials and the normalization condition 


$$
\langle n, \bar{n}, N, \bar{N}, M, \bar{M} \mid n, \bar{n}, N, \bar{N}, M, \bar{M}\rangle=\left(\frac{N_{1} ! N_{2} ! \cdots}{1^{N_{1} 2^{N_{2} \cdots}}}\right)\left(\frac{\bar{N}_{1} ! \bar{N}_{2} ! \cdots}{1^{\bar{N}_{1}} 2^{\bar{N}_{2}} \cdots}\right) .
$$

It is easy to see that $u^{\dagger}=u^{-1}, x_{2 n}^{\dagger}=(1 / n)\left(\partial / \partial x_{2 n}\right), x_{2 n-1}^{\dagger}=\left(\partial / \partial x_{2 n-1}\right)$, and similarly for the adjoint operators of the remaining variables. The vacuum vector is given by $|0\rangle=1$.

The two-point functions for the fields $\Phi$ and $\bar{\Phi}$ are

$$
\begin{gathered}
\left\langle 0\left|T\left[\Phi(Z) \Phi\left(Z^{\prime}\right)\right]\right| 0\right\rangle=\left\langle 0\left|T\left[\bar{\Phi}(Z) \bar{\Phi}\left(Z^{\prime}\right)\right]\right| 0\right\rangle=0, \\
\left\langle 0\left|T\left[\Phi(Z) \bar{\Phi}\left(Z^{\prime}\right)\right]\right| 0\right\rangle=\ln \left(z-z^{\prime}-\alpha \alpha^{\prime}\right) .
\end{gathered}
$$

Hence, for the $B-C$ system we have

$$
\begin{gathered}
\left\langle 0\left|T\left[B(Z) B\left(Z^{\prime}\right)\right]\right| 0\right\rangle=\left\langle 0\left|T\left[C(Z) C\left(Z^{\prime}\right)\right]\right| 0\right\rangle=0, \\
\left\langle 0\left|T\left[B(Z) C\left(Z^{\prime}\right)\right]\right| 0\right\rangle=\frac{\alpha-\alpha^{\prime}}{z-z^{\prime}} .
\end{gathered}
$$

In order to construct solutions of Eq. (24), we introduce the following deformation of the integral kernel of Eq. (24):

$$
a\left(Z, Z^{\prime}, \theta, \mathrm{t}\right)=a\left(z, \alpha+\lambda(z, \theta, \mathrm{t}), z^{\prime}, \alpha^{\prime}+\lambda\left(z^{\prime}, \theta, \mathrm{t}\right)\right)
$$

and the function

$$
F(Z, x, \theta, \mathrm{t}, \overline{\mathrm{t}})=\langle 1|\exp [H(x, \theta, \mathrm{t}, \overline{\mathrm{t}})] T[B(Z) g(\theta, \overline{\mathrm{t}})]| 0\rangle,
$$

where $|1\rangle=u, H(x, \theta, \mathrm{t}, \overline{\mathrm{t}})$ is the operator

$$
\begin{aligned}
H= & x \frac{\partial}{\partial x_{2}}+\sum_{n=1}^{\infty}\left(t_{2 n}+\theta t_{2 n-1}\right) \frac{\partial}{\partial x_{2 n}}+\theta \frac{\partial}{\partial x_{1}}+\sum_{n=1}^{\infty} t_{2 n-1} \frac{\partial}{\partial x_{2 n-1}} \\
& +\sum_{n=1}^{\infty} \bar{t}_{2 n} \frac{\partial}{\partial \bar{x}_{2 n}}+\sum_{n=1}^{\infty} \bar{t}_{2 n-1} \frac{\partial}{\partial \bar{x}_{2 n-1}}
\end{aligned}
$$

and $g(\theta, \overline{\mathrm{t}})$ is the following function valued in the supergroup $\mathrm{GL}(\infty \mid \infty)$ :

$$
g(\theta, \overline{\mathrm{t}})=\exp A(\theta, \bar{t}), \quad A(\theta, \overline{\mathrm{t}})=\frac{1}{\pi} \int d^{2 \mid 1} P d^{2 \mid 1} Q B(P) a(P, Q, \theta, \overline{\mathrm{t}}) C(Q)
$$

At this point we may perform the same analysis as in Sec. II. We use Wick's theorem to expand the operator

$$
T[B(Z) g(\theta, \mathrm{t})]=\sum_{N=0}^{\infty} \frac{1}{N !} T\left[B(Z) A(\theta, \mathrm{t})^{N}\right]
$$

as a series of normal ordered terms with all possible $T$-pairings. Thus, as in the standard KP case, the only nonanalytic terms in $z$ come from the pairings of $B(Z)$ with the operators $C\left(Z^{\prime}\right)$ inside the factors $A(\theta, t)$. Furthermore, we have

$$
\frac{\partial}{\partial \bar{z}}\left\langle 0\left|T\left[B(Z) C\left(Z^{\prime}\right)\right]\right| 0\right\rangle=\pi \delta\left(Z-Z^{\prime}\right),
$$


where $\delta\left(Z-Z^{\prime}\right)=\left(\alpha-\alpha^{\prime}\right) \delta\left(z-z^{\prime}\right)=-\delta\left(Z^{\prime}-Z\right)$ is the distribution verifying

$$
\int d^{2 \mid 1} Z \delta(Z-Q) \varphi(Z)=\varphi(Q)
$$

Therefore, one finds that

$$
\frac{\partial}{\partial \bar{z}} F(Z, x, \theta, \mathrm{t}, \overline{\mathrm{t}})=\int d^{2 \mid 1} Z^{\prime} a\left(Z^{\prime}, Z, \theta, \overline{\mathrm{t}}\right) F\left(Z^{\prime}, x, \theta, t, \overline{\mathrm{t}}\right)
$$

Moreover, from Eqs. (21), (26) it follows that the asymptotic form of $F$ is

$$
F(Z, x, \theta, \mathrm{t}, \overline{\mathrm{t}})=\tau(x, \theta, \mathrm{t}, \overline{\mathrm{t}}-\epsilon(Z)) f_{0}(z, \alpha+\lambda(z, \theta, \overline{\mathrm{t}}), x, \theta, \overline{\mathrm{t}}),
$$

where the function $\tau$ is defined by

$$
\tau(x, \theta, \mathrm{t}, \overline{\mathrm{t}})=\langle 0|\exp [H(x, \theta, \mathrm{t}, \overline{\mathrm{t}})] T[g(\theta, \overline{\mathrm{t}})]| 0\rangle
$$

and $\epsilon(Z)=\left\{\epsilon_{n}(Z)\right\}$ denotes the vector

$$
\epsilon_{2 n}(Z)=\frac{1}{n z^{n}}, \quad \epsilon_{2 n-1}(Z)=-\frac{\alpha}{z^{n}}
$$

As a consequence we conclude that

$$
f(Z, x, \theta, \mathrm{t})=\frac{F(z, \alpha-\lambda(z, \theta, \mathrm{t}), x, \theta, \mathrm{t}, \overline{\mathrm{t}})}{\tau(x, \theta, \mathrm{t}, \overline{\mathrm{t}})}
$$

is a wave function for the SKP hierarchies.

It is worth noticing that the function (31) does not depend on $\bar{t}$. To prove this fact observe that its modified wave function $\hat{f}$ satisfies Eq. (25) and that the integral kernel and the inhomogeneous term in Eq. (25) are independent of $\overline{\mathrm{t}}$. Therefore, the method of successive approximations shows that $\hat{f}$ is also independent of $\bar{t}$.

As $z \rightarrow \infty$ the wave function (31) can be written in terms of the $\tau$-function as

$$
f(Z, x, \theta, \mathrm{t})=f_{0}(Z, x, \theta, \mathrm{t}) \frac{\exp (-\lambda(z, \theta, \mathrm{t})(\partial / \partial \alpha)) \tau(x, \theta, \mathrm{t}, \overline{\mathrm{t}}-\epsilon(Z))}{\tau(x, \theta, \mathrm{t}, \overline{\mathrm{t}})} .
$$

In order to establish bilinear identities for the super-KP hierarchies it is required to introduce the odd function

$$
G(Z, x, \theta, \mathrm{t}, \overline{\mathrm{t}})=\langle-1|\exp [H(x, \theta, \mathrm{t}, \overline{\mathrm{t}})] T[C(Z) g(\theta, \overline{\mathrm{t}})]| 0\rangle,
$$

where $|-1\rangle=u^{-1}$. Then, by repeating the arguments used in the analysis of $F$, we get the following $\bar{\partial}$-equation for $G$ :

$$
\frac{\partial}{\partial \bar{z}} G(Z, x, \theta, \mathrm{t}, \overline{\mathrm{t}})=\int d^{2 \mid 1} Z^{\prime} a\left(Z, Z^{\prime}, \theta, \mathrm{t}\right) G\left(Z^{\prime}, x, \theta, \mathrm{t}, \overline{\mathrm{t}}\right)
$$

Thus, by defining the function

$$
g(Z, x, \theta, \mathrm{t}, \overline{\mathrm{t}})=\frac{G(z, \alpha-\lambda(z, \theta, \overline{\mathrm{t}}), x, \theta, \mathrm{t}, \overline{\mathrm{t}})}{\tau(x, \theta, \mathrm{t}, \overline{\mathrm{t}})}
$$


and taking a circle $\gamma(z \mid=r)$ in the complex plane such that the support of the distribution $a(P, Q)$ lies inside $\gamma \times \gamma$, we get the bilinear identity

$$
\oint_{\gamma} d Z f(Z, x, \theta, \mathrm{t}) g\left(Z, x^{\prime}, \theta^{\prime}, \mathrm{t}^{\prime}, \overline{\mathrm{t}}\right)=0
$$

where $d Z=d z \cdot d \alpha$. The proof of Eq. (35) follows by using Eqs. (28) and (33) in the form

$$
\begin{aligned}
\oint_{\gamma} d Z f(Z, x, \theta, \mathrm{t}) g\left(Z, x^{\prime}, \theta^{\prime}, \mathrm{t}^{\prime}, \overline{\mathrm{t}}^{\prime}\right)= & 2 i \int d^{2 \mid 1} Z \frac{\partial}{\partial \bar{z}}\left[f(Z, x, \theta, \mathrm{t}) g\left(Z, x^{\prime}, \theta^{\prime}, \mathrm{t}^{\prime}, \overline{\mathrm{t}^{\prime}}\right)\right] \\
= & 2 i \int d^{2 \mid 1} Z d^{2 \mid 1} Z^{\prime} f\left(Z^{\prime}, x, \theta, \mathrm{t}\right) a\left(Z^{\prime}, Z\right) g\left(Z, x^{\prime}, \theta^{\prime}, \mathrm{t}^{\prime}, \overline{\mathrm{t}^{\prime}}\right) \\
& +2 i \int d^{2 \mid 1} Z d^{2 \mid 1} Z^{\prime} f(Z, x, \theta, \mathrm{t}) a\left(Z, Z^{\prime}\right) g\left(Z^{\prime}, x^{\prime}, \theta^{\prime}, \mathrm{t}^{\prime}, \overline{\mathrm{t}^{\prime}}\right) \\
= & 0 .
\end{aligned}
$$

The asymptotic form of $G$ as $z \rightarrow \infty$ is

$$
\begin{aligned}
G(Z, x, \theta, \mathrm{t}, \overline{\mathrm{t}})= & {\left[f_{0}(z, \alpha+\lambda, x, \theta, \mathrm{t})\right]^{-1}\langle 0|\left(\omega(Z, \overline{\mathrm{t}})+P\left(Z, \partial_{\mathrm{x}}\right)\right) } \\
& \times \exp [H(x, \theta, \mathrm{t}, \overline{\mathrm{t}}+\epsilon(Z))] T[g(\theta, \mathrm{t})]|0\rangle,
\end{aligned}
$$

where

$$
\omega(Z, \overline{\mathrm{t}})=\sum_{n=1}^{\infty} z^{n-1}\left(\bar{t}_{2 n-1}+n \alpha \bar{t}_{2 n}\right), \quad P\left(Z, \partial_{\mathrm{x}}\right)=\sum_{n=1}^{\infty} \frac{1}{z^{n}} \frac{\partial}{\partial x_{2 n-1}}+\alpha \sum_{n=1}^{\infty} \frac{1}{z^{n+1}} \frac{\partial}{\partial x_{2 n}} .
$$

Hence, taking into account that

$$
\frac{\partial}{\partial x_{2 n}} \cdot e^{H}=\frac{\partial e^{H}}{\partial t_{2 n}}, \quad \frac{\partial}{\partial x_{2 n-1}} \cdot e^{H}=\frac{\partial e^{H}}{\partial t_{2 n-1}}+\theta \frac{\partial e^{H}}{\partial t_{2 n}}
$$

we get

$$
\begin{aligned}
& \left\langle 0\left|P\left(Z, \partial_{\mathrm{x}}\right) \cdot \exp [H(x, \theta, \mathrm{t}, \overline{\mathrm{t}}+\epsilon(Z))] T[g(\theta, \mathrm{t})]\right| 0\right\rangle \\
& \quad=Q\left(Z, \partial_{\mathrm{t}}\right) \tau(x, \theta, \mathrm{t}, \overline{\mathrm{t}}+\epsilon(Z))-\left\langle 0\left|\exp [H(x, \theta, \mathrm{t}, \overline{\mathrm{t}}+\epsilon(Z))] Q\left(Z, \partial_{\mathrm{t}}\right) T[g(\theta, \mathrm{t})]\right| 0\right\rangle,
\end{aligned}
$$

where

$$
Q\left(Z, \partial_{t}\right)=\sum_{n=1}^{\infty} \frac{1}{z^{n}}\left(\frac{\partial}{\partial t_{2 n-1}}+\theta \frac{\partial}{\partial t_{2 n}}\right)+\alpha \sum_{n=1}^{\infty} \frac{1}{z^{n+1}} \frac{\partial}{\partial t_{2 n}}
$$

The second term in the right-hand side of Eq. (37) vanishes for the JKP hierarchy since $\lambda$, and consequently $g(\theta, t)$, is independent of $t$. Therefore, the asymptotic form of the function (34) is

$$
g(Z, x, \theta, \mathrm{t}, \overline{\mathrm{t}})=\frac{\exp (-z \theta \cdot(\partial / \partial \alpha))\left[\left(\omega(Z, \overline{\mathrm{t}})+Q\left(Z, \partial_{\mathrm{t}}\right)\right) \tau(x, \theta, \mathrm{t}, \overline{\mathrm{t}}+\epsilon(Z))\right]}{f_{0}(Z, x, \theta, \mathrm{t}) \tau(x, \theta, \mathrm{t}, \overline{\mathrm{t}})} .
$$


Thus, we may use this expression together with Eq. (32) to rewrite the bilinear identity (35) of the JKP hierarchy as a constraint for the $\tau$-function (30). Nevertheless, for the MRKP hierarchy the explicit dependence of $\lambda$ on the variables $t$ makes the function (34) as well as the bilinear identity (35) depend on additional correlation functions apart from (30).

Explicit solutions to the super-KP hierarchics arc obtained from some particular distributions $a(P, Q)$. For example, the generalization of the $N$-soliton solution corresponds to the choice

$$
a(P, Q)=-\pi \sum_{j=1}^{N} \omega_{j} \delta\left(P-P_{j}\right) \delta\left(Q-Q_{j}\right)
$$

where $P_{j}=\left(P_{j}, \beta_{j}\right)$ and $Q_{j}=\left(q_{j}, \nu_{j}\right)$. Thus

$$
g(\theta, \mathrm{t})=\sum_{I}(-1)^{(\# I)(\# I-1) / 2} \prod_{j \in I} \omega_{j} \prod_{j \in I} B\left(p_{j}, \beta_{j}-\lambda\left(p_{j}, \theta, \mathrm{t}\right)\right) C\left(q_{j}, \nu_{j}-\lambda\left(q_{j}, \theta, \mathrm{t}\right)\right),
$$

where $I$ runs over the subsets of $\{1, \ldots, N\}$. Assuming $\left|p_{1}\right|>\left|q_{1}\right|>\cdots>\left|p_{N}\right|>\left|q_{N}\right|$ we find

$$
\begin{aligned}
T\left[\prod_{j \in I} B\left(P_{j}\right) C\left(Q_{j}\right)\right]= & \prod_{j \in I} \exp \left[\sum_{n=1}^{\infty} p_{j}^{n} x_{2 n}+\beta_{j} \sum_{n=1}^{\infty} p_{j}^{n-1} x_{2 n-1}\right] \\
& \times \exp \left[-\sum_{n=1}^{\infty} q_{j}^{n} x_{2 n}-\nu_{j} \sum_{n=1}^{\infty} q_{j}^{n-1} x_{2 n-1}\right] \prod_{j \in I}\left\{\sum_{n=1}^{\infty} q_{j}^{n-1} \bar{x}_{2 n-1}\right. \\
& +\nu_{j} \sum_{n=1}^{\infty} n q_{j}^{n-1} \bar{x}_{2 n}+\frac{\nu_{j}}{q_{j}} u \frac{\partial}{\partial u}+\nu_{j} \sum_{n=1}^{\infty} \frac{1}{q_{j}^{n+1}} \frac{\partial}{\partial x_{2 n}} \\
& \left.+\sum_{n=1}^{\infty} \frac{1}{q_{j}^{n}} \frac{\partial}{\partial x_{2 n}-1}+\sum_{i \in I} \frac{\beta_{i}-\nu_{j}}{p_{i}-q_{j}}-\sum_{i \neq j} \frac{\nu_{i}-\nu_{j}}{q_{i}-q_{j}}\right\} \\
& \times \prod_{j \in I}\left(\frac{p_{j}}{q_{j}}\right)^{\bar{u} \partial_{\bar{u}}}\left[\exp -\sum_{n=1}^{\infty} \frac{1}{n p_{j}^{n}} \frac{\partial}{\partial \bar{x}_{2 n}}+\beta_{j} \sum_{n=1}^{\infty} \frac{1}{p_{j}^{n}} \frac{\partial}{\partial \bar{x}_{2 n-1}}\right] \\
& \times \exp \left[\sum_{n=1}^{\infty} \frac{1}{n q_{j}^{n}} \frac{\partial}{\partial \bar{x}_{2 n}}-\nu_{j} \sum_{n=1}^{\infty} \frac{1}{q_{j}^{n}} \frac{\partial}{\partial \bar{x}_{2 n-1}}\right]
\end{aligned}
$$

so that

$$
\begin{aligned}
\tau(x, \theta, \mathrm{t}, \overline{\mathrm{t}})= & \sum_{I}(-1)^{(\# I)(\# I-1) / 2} \prod_{j \in I} \omega_{j} \prod_{j \in I}\left\{\sum_{n=1}^{\infty} q_{j}^{n-1} \bar{t}_{2 n-1}+\left(\nu_{j}-\lambda\left(q_{j}, \theta, t\right)\right) \sum_{n=1}^{\infty} n q_{j}^{n-1} \bar{t}_{2 n}\right. \\
& \left.+\sum_{i \in I} \frac{\beta_{i}-\lambda\left(p_{i}, \theta, t\right)+\lambda\left(q_{j}, \theta, t\right)-\nu_{j}}{p_{i}-q_{j}}-\sum_{i \neq j} \frac{\nu_{i}-\lambda\left(q_{i}, \theta, t\right)+\lambda\left(q_{j}, \theta, t\right)-\nu_{j}}{q_{i}-q_{j}}\right\} \\
& \times \prod_{j \in I} f_{0}\left(P_{j}, x, \theta, \mathrm{t}\right) f_{0}^{-1}\left(Q_{j}, x, \theta, \mathrm{t}\right) .
\end{aligned}
$$

In particular, the expression of the one-soliton solution is

$$
\tau(x, \theta, \mathrm{t}, \overline{\mathrm{t}})=1+\omega_{1}\left\{\sum_{n=1}^{\infty} q_{1}^{n-1} \bar{t}_{2 n-1}+\left(\nu_{1}-\lambda\left(q_{1}, \theta, \mathrm{t}\right)\right) \sum_{n=1}^{\infty} n q_{1}^{n-1} \bar{t}_{2 n}\right.
$$




$$
\left.+\frac{\beta_{1}-\lambda\left(p_{1}, \theta, \mathrm{t}\right)+\lambda\left(q_{1}, \theta, \mathrm{t}\right)-\nu_{1}}{p_{1}-q_{1}}\right\} f_{0}\left(P_{1}, x, \theta, \mathrm{t}\right) f_{0}^{-1}\left(Q_{1}, x, \theta, \mathrm{t}\right)
$$

We can construct rational solutions by taking distributions of the form

$$
a(P, Q)=\frac{\pi \omega}{(2 \pi i)^{2}}\left(\beta-\beta_{0}\right) \delta_{\gamma}(p)\left(\nu-\nu_{0}\right) \delta_{\gamma}(q) \frac{1}{p^{n+1} q^{m+1}}, \quad n, m \geqslant 0,
$$

where $P=(p, \beta), Q=(q, \nu)$ and $\delta_{\gamma}$ is the delta function with support on a circle $\gamma(|z|=r)$ such that

$$
\int d^{2} z \delta_{\gamma}(z) \phi(z)=\oint_{\gamma} d z \phi(z)
$$

To determine the corresponding wave function we insert Eq. (38) in the $\bar{\partial}$-equation (25) satisfied by $\hat{f}$, and taking into account that $z \rightarrow \infty$ we find

$$
\begin{aligned}
\hat{f}(Z, x, \theta, \mathrm{t})= & -\omega\left\{\sum_{k=0}^{m} \frac{1}{z^{k+1}}\left[\left(\alpha-\nu_{0}\right) \tilde{p}\left(\nu_{0}, x, \theta, \mathrm{t}\right)+\sum_{r=1}^{m-k} \lambda_{r} \tilde{p}_{m-k-r}\left(\nu_{0}, x, \theta, \mathrm{t}\right)\right]\right\} \\
& \times\left\{\frac{1}{2 \pi i} \oint_{\gamma} \frac{d z^{\prime \prime}}{z^{n+1}} f\left(z^{\prime \prime}, \beta_{0}, x, \theta, \mathrm{t}\right)\right\},
\end{aligned}
$$

where the polynomials $p_{l}(\alpha, x, \theta, \mathrm{t}), \tilde{p}(\alpha, x, \theta, \mathrm{t})$, and $\lambda_{r}(\theta, \mathrm{t})$ are defined by

$$
\begin{gathered}
f_{0}(Z, x, \theta, \mathrm{t})=\sum_{l=0}^{\infty} p_{l}(\alpha, x, \theta, \mathrm{t}) z^{l}, \\
f_{0}^{-1}(Z, x, \theta, \mathrm{t})=\sum_{l=0}^{\infty} \tilde{p}_{l}(\alpha, x, \theta, \mathrm{t}) z^{l}, \quad \lambda(z, \theta, t)=\sum_{r=1}^{\infty} \lambda_{r}(\theta, \mathrm{t}) z^{r} .
\end{gathered}
$$

Now, Eq. (39) implies

$$
\hat{f}(Z, x, \theta, \mathrm{t})=1+\sum_{j=1}^{m+1} \frac{w_{2 j}(x, \theta, \mathrm{t})+\alpha w_{2 j-1}(x, \theta, \mathrm{t})}{z^{j}} .
$$

Then, introducing Eq. (40) in Eq. (39) it follows that $\omega w_{i}=0 i=1, \ldots, 2 m+2$, so that

$$
\frac{\omega}{2 \pi i} \oint_{\gamma} \frac{d z^{\prime \prime}}{z^{\prime \prime n+1}} f\left(z^{\prime \prime}, \beta_{0}, x, \theta, \mathrm{t}\right)=\omega p_{n}\left(\beta_{0}, x, \theta, \mathrm{t}\right)
$$

and

$$
\begin{aligned}
\hat{f}(Z, x, \theta, \mathrm{t})= & 1-\omega p_{n}\left(\beta_{0}, x, \theta, \mathrm{t}\right) \sum_{k=0}^{\infty} \frac{1}{z^{k+1}}\left[\left(\alpha-\nu_{0}\right) \tilde{p}_{m-k}\left(\nu_{0}, x, \theta, \mathrm{t}\right)\right. \\
& \left.+\sum_{r=1}^{m-k} \lambda_{r} \tilde{p}_{m-k-r}\left(\nu_{0}, x, \theta, \mathrm{t}\right)\right] .
\end{aligned}
$$

In particular, for the JKP hierarchy $\lambda_{1}=\theta, \lambda_{r}=0, r>1$, so that we get 


$$
\begin{aligned}
f(Z, x, \theta, \mathrm{t})= & f_{0}(Z, x, \theta, \mathrm{t})\left\{1-\omega p_{n}\left(\beta_{0}, x, \theta, \mathrm{t}\right) \sum_{k=0}^{m} \frac{1}{z^{k+1}}\left[\left(\theta \tilde{p}_{m-k-r}\left(\nu_{0}, x, \theta, \mathrm{t}\right)\right.\right.\right. \\
& \left.\left.\left.-\nu_{0} \tilde{p}_{m-k}\left(\nu_{0}, x, \theta, \mathrm{t}\right)\right)+\left(\alpha-\lambda(z, \theta, \mathrm{t}) \tilde{p}_{m-k}\left(\nu_{0}, x, \theta, \mathrm{t}\right)\right)\right]\right\}
\end{aligned}
$$

and for the MRKP hierarchy $\lambda_{1}=\theta-t_{1}, \lambda_{r}=-t_{2 r-1}, r>1$

$$
\begin{aligned}
f(Z, x, \theta, \mathrm{t})= & f_{0}(Z, x, \theta, \mathrm{t})\left\{1-\omega p_{n}\left(\beta_{0}, x, \theta, \mathrm{t}\right) \sum_{k=0}^{m} \frac{1}{z^{k+1}}\left[\left(-\nu_{0} \tilde{p}_{m-k}\left(\nu_{0}, x, \theta, \mathrm{t}\right)\right.\right.\right. \\
& \left.+\left(\theta-t_{1}\right) \tilde{p}_{m-k-r}\left(\nu_{0}, x, \theta, \mathrm{t}\right)-\sum_{r=2}^{m-k} t_{2 r-1} \tilde{p}_{m-k-r}\left(\nu_{0}, x, \theta, \mathrm{t}\right)\right) \\
& \left.\left.+\left(\alpha-\lambda(z, \theta, \mathrm{t}) \tilde{p}_{m-k}\left(\nu_{0}, x, \theta, \mathrm{t}\right)\right)\right]\right\} .
\end{aligned}
$$

The first few $p_{l}(\alpha, x, \theta, \mathrm{t}), \tilde{p}_{l}(\alpha, x, \theta, \mathrm{t})$ are given by

$$
\begin{gathered}
p_{0}(\alpha, x, \theta, \mathrm{t})=1+\alpha\left(\theta+t_{1}\right), \\
p_{1}(\alpha, x, \theta, \mathrm{t})=\left(x+t_{2}\right)\left[1+\alpha\left(\theta+t_{1}\right)\right]+\alpha t_{3}-r \theta t_{1}, \\
p_{2}(\alpha, x, \theta, \mathrm{t})=\left[t_{4}+\frac{\left(x+t_{2}\right)^{2}}{2}\right]\left[1+\alpha\left(\theta+t_{1}\right)\right]+\left(x+t_{2}\right)\left(\alpha t_{3}-r \theta t_{1}\right)+\alpha t_{5}-r \theta t_{3}, \\
\tilde{p}_{0}(\alpha, x, \theta, \mathrm{t})=1-\alpha\left(\theta+t_{1}\right), \\
\tilde{p}_{1}(\alpha, x, \theta, \mathrm{t})=-\left(x+t_{2}\right)\left[1-\alpha\left(\theta+t_{1}\right)\right]-\alpha t_{3}+r \theta t_{1}, \\
\tilde{p}_{2}(\alpha, x, \theta, \mathrm{t})=\left[-t_{4}+\frac{\left(x+t_{2}\right)^{2}}{2}\right]\left[1-\alpha\left(\theta+t_{1}\right)\right]+\left(x+t_{2}\right)\left(\alpha t_{3}-r \theta t_{1}\right)-\alpha t_{5}+r \theta t_{3},
\end{gathered}
$$

where $r=1$ for the MRKP and $r=0$ for the JKP.

\section{ACKNOWLEDGMENTS}

The authors would like to thank Professor A. Ibort for many helpful conversations. The financial support of the CICYT is also acknowledged.

\footnotetext{
${ }^{1}$ E. Date, M. Jimbo, M. Kashiwara, and T. Miwa, "Transformation groups for soliton equations," in Nonlinear Integrable Systems-Classical and Quantum Theory, edited by M. Jimbo and T. Miwa (World Scientific, Singapore, 1983). ${ }^{2}$ M. R. Douglas, Phys. Lett. B 238, 176 (1990).

${ }^{3}$ R. Dijkgraff, H. Verlinde, and E. Verlinde, Nucl. Phys. B 348, 435 (1991).

${ }^{4}$ Yu I. Manin and A. O. Radul, Commun. Math. Phys. 98, 65 (1985).

${ }^{5}$ M. Mulase, J. Diff. Geom. 34, 651 (1991).

${ }^{6}$ J. M. Rabin, Commun. Math. Phys. 137, 533 (1991).

${ }^{7}$ L. Alvarez-Gaumé, H. Itoyama, J. L. Mañes, and A. Zadra, Int. J. Mod. Phys. A 7, 5337 (1992).

${ }^{8}$ L. Alvarez-Gaumé, K. Becker, M. Becker, R. Emperan, and J. Mañes, Int. J. Mod. Phys. A 8, 2297 (1993).

${ }^{9}$ K. Becker and M. Becker, Mod. Phys. Lett. A 8, 1205 (1993).

${ }^{10}$ M. Mañas, L. Martinez Alonso, and E. Medina, Phys. Lett. B 336, 178 (1994).

ItE. J. Martinec and G. M. Sotkov, Phys. Lett. B 208, 249 (1988).

12 A. LeClair, Nucl. Phys. B 314, 425 (1989).
} 
${ }^{13}$ R. Beals and R. R. Coifman, Physica D 18, 242 (1986).

${ }_{14}$ M. J. Ablowitz, D. Bar Jacob, and A. S. Fokas, Stud. Appl. Math. 69, 135 (1983).

${ }^{15}$ M. Jaulent, M. Manna, and L. Martinez Alonso, Inverse Problems 4, 123 (1988).

${ }^{16} \mathrm{~K}$. Ueno, H. Yamada, and K. Ikeda, Commun. Math. Phys. 124, 57 (1989). 
Journal of Mathematical Physics is copyrighted by AIP Publishing LLC (AIP). Reuse of AIP content is subject to the terms at: http://scitation.aip.org/termsconditions. For more information, see http://publishing.aip.org/authors/rights-and-permissions. 